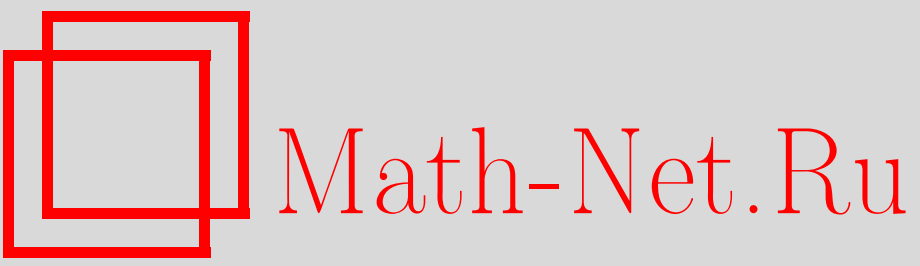

А. Ю. Каменщик, Н. А. Свешников, Инфракрасные расходимости и асимптотические состояния кварков в квантовой хромодинамике, ТМФ, 1998, том 117, номер 2, 175-188

DOI: https://doi.org/10.4213/tmf925

Использование Общероссийского математического портала Math-Net.Ru подразумевает, что вы прочитали и согласны с пользовательским соглашением

http://www.mathnet.ru/rus/agreement

Параметры загрузки:

IP: 54.147 .182 .235

26 апреля 2023 г., 16:10:55 
ТЕОРЕТИЧЕСКАЯ

И МАТЕМАТИЧЕСКАЯ

ФИЗИКА

Том 117, № 2

ноябрь, 1998

\section{ИНФРАКРАСНЫЕ РАСХОДИМОСТИ И АСИМПТОТИЧЕСКИЕ СОСТОЯНИЯ КВАРКОВ В КВАНТОВОЙ ХРОМОДИНАМИКЕ}

Показано, что с помощью метода асимптотической динамики невозможно построить асимптотические состояния свободных кварков в квантовой хромодинамике.

В 1983 г. Никита Алексеевич Свешников и я опубликовали статью [1] об отсутствии свободных кварков в пертурбативной квантовой хромодинамике (КХД). В статье был представлен лишь набросок строгого доказательства соответствуюших утверждений. Мы планировали в дальнейшем опубликовать подробную статью, посвяшенную этой теме, но не сделали этого. Никита Алексеевич всегда стремился к высокому уровню математической строгости и добивался его в своих опубликованных статьях. Сейчас, когда его нет с нами, я не могу представить текст того качества, который, как я понимаю, мог бы удовлетворить его высоким критериям. Тем не менее я решился опубликовать наши старые заметки в надежде, что они могут быть полезны людям, занимаюшимся проблемами инфракрасных расходимостей и конфайнмента в КХД. При этом я должен подчеркнуть, что все идеи этой работы принадлежат Никите Алексеевичу, а вся ответственность за недостатки изложения должна быть отнесена на мой счет.

А.Ю. Каменщик

\section{1. ВВЕДЕНИЕ. ПРОБЛЕМА УДЕРЖАНИЯ КВАРКОВ И ИНФРАКРАСНЫЕ РАСХОДИМОСТИ}

КХД стала главным кандидатом на роль фундаментальной теории сильных взаимодействий. Однако свободные кварки не были обнаружены экспериментально [2], поэтому возникла гипотеза об удержании кварков ("конфайнмент") [3]. Суть этой гипотезы сводится к тому, что сушествуют только связные бесцветные состояния кварков - адроны. Свободных же кварков, равно как и иных цветных состояний, в природе нет.

Имеется множество подходов к теоретическому обоснованию этой гипотезы [4]. Наряду с полуфеноменологическими подходами также широко распространены исследования инфракрасной структуры теории, опираюшиеся на КХД “в чистом виде", т.е. на лагранжиан КХ Д, и непосредственно следуюшие из него свойства функций Грина и матричных элементов. Среди них можно выделить решение уравнений Швингера-Дайсона

\footnotetext{
${ }^{*}$ Институт теоретической физики им. Л. Д. Ландау РАН, Москва, Россия
} 
в инфракрасном пределе КХ Д и исследование инфракрасных функций Грина [5], с одной стороны, и исследование инфракрасных расходимостей матричных элементов и сечений в рамках теории возмущений, с другой [6-12]. Имелись также попытки применить метод асимптотической динамики [13] к исследованию сокращения инфракрасных расходимостей в матричных элементах [14] и к проблеме конфайнмента [15].

Среди работ, посвяшенных изучению инфракрасных расходимостей матричных элементов, для нас особенно интересны статьи $[7,8,12]$. В работе [12] с помощью анализа фейнмановских диаграмм были выделены главные инфракрасные расходимости и доказана их "экспоненциация", аналогичная электродинамической [16]. При этом получившийся в экспоненте фактор обрашал в нуль любые несинглетные по цвету состояния. В статьях $[7,8]$ проблема невылетания кварков не рассматривалась, но был произведен тщательный анализ инфракрасных расходимостей, результаты которого использованы в нашей статье [1]. Уравнения, полученные в работах $[7,8]$ для главных инфракрасных расходимостей матричных элементов с произвольным числом внешних концов, соответствующих массивным кваркам, выглядят следующим образом:

$$
\begin{aligned}
& \frac{\partial}{\partial \lambda} T_{i_{1} \ldots i_{N}}\left(p_{1}, \ldots, p_{N}\right)=-\frac{1}{1-\frac{22}{3} C_{A} \frac{\lambda}{4 \pi}} \sum_{\alpha, \beta=1, \alpha \neq \beta}^{N} \eta_{\alpha} \eta_{\beta} \lambda_{i_{\alpha} j_{\alpha}}^{a} \lambda_{i_{\beta} j_{\beta}}^{a} \times \\
& \times F\left(\left(p_{\alpha}+p_{\beta}\right)^{2}\right) T_{i_{1} \ldots j_{\alpha} \ldots j_{\beta} \ldots i_{N}}\left(p_{1}, \ldots, p_{N}\right) \\
& \lim _{\lambda \rightarrow 0} T_{i_{1} \ldots i_{N}}\left(p_{1}, \ldots, p_{N}\right)=T_{i_{1} \ldots i_{N}}^{0}\left(p_{1}, \ldots, p_{N}\right)
\end{aligned}
$$

где $\lambda=g^{2} / 2 \epsilon, \epsilon=(d-4) / 2, d$ - размерность пространства-времени, $T_{i_{1} \ldots i_{N}}\left(p_{1}, \ldots, p_{N}\right)$ - главная инфракрасно расходяшаяся часть перенормированной амплитуды процесса, содержашей $N$ внешних кварковых линий с импульсами $p_{\alpha}$ и цветными индексами $i_{\alpha}$, $\eta_{\alpha}-$ знаковьй фактор, равный +1 для выходящей линии и -1 для входящей, $\lambda^{a}-$ генераторы цветной группы $G$,

$$
F\left(p^{2}\right)=\int_{0}^{1} d y \frac{p^{2}-2 m^{2}}{m^{2}-p^{2}(1-y) y-i 0}+2,
$$

$C_{A}$ - собственное значение квадратичного оператора Казимира группы $G$ в присоединенном представлении, $T_{i_{1} \ldots i_{N}}^{0}\left(p_{1}, \ldots, p_{N}\right)$ - простейшая диаграмма с тем же набором внешних импульсов и цветных индексов, что и $T_{i_{1} \ldots i_{N}}\left(p_{1}, \ldots, p_{N}\right)$.

В работе [8] было показано, что учет неглавных расходимостей не приводит к существенному изменению уравнений Киношиты-Укавы (1.1), (1.2).

Говоря об исследовании инфракрасных расходимостей в КХД, следует упомянуть работы [10], посвяшенные проверке теоремы Киношиты, Ли и Науэнберга [17] на уровне теории возмушений. Как и следовало ожидать, эта обшая теорема выполняется, в отличие от программы Блоха-Нордсика [18], которая реализуется лишш для рассеяния одного кварка на синглетном по цвету потенциале [19].

Особый интерес представляют работы по применению метода асимптотической динамики [13] к КХД. Все эти работы основаны на оценке различных членов оператора асимптотической эволюции, расписанного с помошью формулы Магнуса [20] для 
$T$-экспоненты, и удержании первых нескольких членов (в отличие от квантовой электродинамики (КЭД) формула Магнуса не обрывается на конечном шаге). Получаемые с помощью такого “обрезанного” оператора асимптотической эволюции асимптотические состояния можно затем подставить в матричные элементы вместо фоковских. При этом инфракрасные расходимости, эффективно учтенные с помощью такого обрезанного оператора, сокрашаются. Однако в более высоких порядках теории возмушений появляются другие расходимости, для сокрашения которых нужно иметь более точное описание асимптотических состояний.

Попытка объяснить конфайнмент с помощью метода асимптотической динамики была предпринята в работе [15], где было высказано интересное предположение, что асимптотические состояния в КХД не могут быть построены из фоковских в отличие от КЭД. Однако это предположение не было доказано.

Существенным шагом в исследовании проблемы конфайнмента было исследование проблемы самосогласованности задачи рассеяния в моделях с неабелевой симметрией, предпринятое в работах Н. А. Свешникова [21-23]. Разработанный в этих работах формализм, будучи применен к КХД [1], приводит к новому результату: если в КХД с массивными кварками и сушествуют физические кварки, то они не могут быть получены из затравочных кварков по теории возмушений. Для доказательства этого факта наряду с методом асимптотической динамики использовались и уравнения Киношиты-Укавы. В последующих разделах будут приведены некоторые детали доказательства, которое было кратко изложено в [1].

\section{2. ФОРМУЛИРОВКА МЕТОДА АСИМПТОТИЧЕСКОЙ ДИНАМИКИ ДЛЯ КХД}

В теории возмущений для определения $S$-матрищы необходимо введение инфракрасной регуляризации. Удобно воспользоваться размерной регуляризацией $[24](\epsilon=(d-$ $4) / 2>0)$. Пусть $S_{\epsilon}$ - регуляризованная матрица рассеяния, соответствуюшая полному $H$ и свободному $H_{0}$ гамильтонианам. Рецепт устранения инфракрасных расходимостей состоит в переходе от представления взаимодействия к представлению асимптотической динамики [13], в котором роль свободного гамильтониана играет асимптотический гамильтониан $H_{\mathrm{as}}=H_{0}+H_{\mathrm{int}}^{\mathrm{as}}$. Здесь $H_{\mathrm{int}}^{\mathrm{as}}-$ часть гамильтониана взаимодействия $H_{\text {int }}=H-H_{0}$, описываюшая процессы с передачей энергии порядка $\Delta \ll m$, где $m-$ масса кварка.

$S$-матрица представления взаимодействия может быть записана в виде

$$
S_{\epsilon}=V_{\epsilon}^{\dagger+} S_{\epsilon}^{\text {as }} V_{\epsilon}^{-},
$$

где асимптотическая $S$-матрица $S_{\epsilon}^{\text {as }}$ и операторы асимптотической эволюции равны соответственно

$$
\begin{aligned}
S_{\epsilon}^{\mathrm{as}} & =\lim _{t_{ \pm} \rightarrow \pm \infty} e^{i H_{\mathrm{as}} t_{+}} e^{-i H\left(t_{+}-t_{-}\right)} e^{-i H_{\mathrm{as}} t_{-}}, \\
V_{\epsilon}^{ \pm} & =\lim _{t \rightarrow \pm \infty} e^{i H_{\mathrm{as}} t} e^{-i H_{0} t},
\end{aligned}
$$

значок $\dagger$ означает эрмитово сопряжение. При наличии инфракрасной регуляризации все операторы, фигурируюшие в $(2.1),(2.2)$, сушествуют и унитарны в рамках теории возмущений. 
Асимптотический гамильтониан КХД содержит кварковые операторы лишш в комбинациях $\rho^{a}(\vec{p})$ - операторов плотности цветных зарядов $[14,15]$, где

$$
\rho^{a}(\vec{p})=b_{i}^{+}(\vec{p}) \lambda_{i j}^{a} b_{j}(\vec{p})-d_{i}^{+}(\bar{p}) \lambda_{i j}^{a} d_{j}(\bar{p}),
$$

$b^{+}, b$ и $d^{+}, d-$ операторы рождения и уничтожения кварков, соответственно.

Легко проверить, что

$$
\left[V_{\epsilon}^{ \pm}, \rho(\vec{p})\right]=0
$$

где

$$
\rho(\vec{p})=b_{i}^{+}(\vec{p}) b_{j}(\vec{p})+d_{i}^{+}(\vec{p}) d_{j}(\vec{p})
$$

есть оператор плотности числа кварков и антикварков. Отсюда следует, что инфракрасно-одетое кварковое состояние

$$
V_{\epsilon}^{ \pm}\left|\Psi^{a}\right\rangle_{q} \otimes|0\rangle_{g}
$$

где $\left|\Psi^{a}\right\rangle_{q}$ - фоковское кварковое состояние с цветным мультииндексом $a,|0\rangle_{g}$ - фоковский вакуум глюонного поля, может быть представлено в виде

$$
\sum_{a^{\prime}}\left|\Psi^{a^{\prime}}\right\rangle_{q} \otimes V_{\epsilon ; a a^{\prime}}^{ \pm}|0\rangle_{g}
$$

где $V_{\epsilon ; a a^{\prime}}^{ \pm}$содержит лишь глюонные операторы и генераторы цветной группы $G$.

Мы предположим, что при снятии регуляризации состояние (2.7) становится асимптотическим состоянием КХД (где $V_{\epsilon ; a a^{\prime}}^{ \pm}|0\rangle_{g}$ играет роль вакуума) и что $S$-матрица в асимптотическом представлении (2.1) не содержит инфракрасных расходимостей по построению. Высказанные предположения эквивалентны утверждению, что в КХД с помошью метода асимптотической динамики можно устранить инфракрасные расходимости из $S$-матрицы. Это утверждение вполне согласуется с рассмотренными во введении результатами анализа инфракрасных расходимостей.

Действительно, рассмотрим вероятность перехода меж ду чисто кварковыми состояниями:

$$
W_{f, i}=\operatorname{Tr}\left(P_{f} S_{\epsilon} P_{i} S_{\epsilon}^{\dagger}\right)
$$

где

$$
P_{f, i}=\left|\Psi_{f, i}\right\rangle_{q}\left\langle\left.\Psi_{f, i}\right|_{q} \otimes \mid 0\right\rangle_{g}\left\langle 0_{g}\right| .
$$

Подстановка (2.1) в (2.8) дает с использованием (2.7) после суммирования по конечным состояниям, которые отличаются энергией, не превышаюшей $\delta E$, следуюшее выражение для величин типа инклюзивных сечений:

$$
\sigma_{i}(\delta E)=\sum_{a^{\prime} a^{\prime \prime}}\left\langle\left. 0\right|_{g} V_{\epsilon ; a^{\prime} a}^{\dagger-} \otimes\left\langle\left.\Psi_{i}^{a^{\prime}}\right|_{q} S_{\epsilon}^{\dagger \mathrm{as}} P_{f}^{\delta E} S_{\epsilon}^{\mathrm{as}} \mid \Psi_{i}^{a^{\prime \prime}}\right\rangle_{q} \otimes V_{\epsilon ; a a^{\prime \prime}}^{-} \mid 0\right\rangle_{g} .
$$

В самом деле, имеем условие

$$
\sum_{f} V_{\epsilon}^{+} P_{f} V_{\epsilon}^{\dagger+}=\sum_{f} P_{f} \equiv P_{f}^{\delta E}
$$


во всех порядках теории возмушений, меньших $\delta E / \Delta$. В силу произвольности $\Delta$ можно гарантировать вьполнение этого условия с точностью до любого наперед заданного порядка.

Суммирование (2.10) по начальным состояниям с разностью энергий $\delta E$ приводит к равенству

$$
\sigma(\delta E)=\operatorname{Tr}\left(P_{f}^{\delta E} S_{\epsilon}^{\mathrm{as}} P_{i}^{\delta E} S_{\epsilon}^{\dagger a s}\right) .
$$

Это выражение не содержит инфракрасных расходимостей в полном соответствии с теоремой Киношиты, Ли и Науэнберга [17].

Рассмотрим теперь вопрос о выполнении программы Блоха-Нордсика [18] в КХД, изучавшийся в статье [19]. Наличие в начальном состоянии лишь одной цветной частицы означает в силу закона сохранения цветного заряда, что оператор

$$
A^{a^{\prime} a^{\prime \prime}}=\left\langle\left.\Psi_{i}^{a^{\prime}}\right|_{q} S_{\epsilon}^{\dagger a s} P_{f}^{\delta E} S_{\epsilon}^{\text {as }} \mid \Psi_{i}^{a^{\prime \prime}}\right\rangle_{q}
$$

пропорционален $\delta^{a^{\prime} a^{\prime \prime}}$. Если бы было несколько частиц, то закон сохранения цветного заряда не привел бы к совпадению мультииндексов. Вследствие этого выражение (2.10) не содержит инфракрасных расходимостей, т.к. произведение

$$
V_{\epsilon ; a^{\prime} a}^{\dagger-} V_{\epsilon ; a a^{\prime \prime}}^{-}{a^{a^{\prime}} a^{\prime \prime}}^{\prime \prime} V_{\epsilon ; a^{\prime} a}^{\dagger-} V_{\epsilon ; a a^{\prime}}^{-}
$$

не содержит полюсов по $\epsilon$ в силу унитарности операторов $V^{ \pm}$, которая сохраняется при снятии регуляризации. Этот результат совпадает с результатом работы [19]. В случае абелевой группы симметрии (КЭД) он обобшается на произвольное начальное состояние электронов и позитронов, поскольку сумма по мультииндексам в (2.10) содержит теперь лишь одно слагаемое.

Однако в случае рассеяния нескольких цветных кварков оператор $V_{\epsilon ; a^{\prime} a}^{\dagger-} V_{\epsilon ; a a^{\prime \prime}}^{-}$уже не является просто произведением унитарного оператора на эрмитово сопряженный $\mathrm{K}$ нему, т.е. тождественным оператором. Компенсации инфракрасных полюсов $1 / \epsilon$, вообще говоря, не происходит. В этом случае усреднение по цветам кварков в начальном состоянии позволяет сделать заключение лишь о сокрашении главных инфракрасных расходимостей. Действительно, после суммирования по $a$ функция $\sigma_{i}(\delta E)$ может быть записана в виде

$$
\begin{aligned}
\overline{\sigma_{i}(\delta E)} & =\sum_{a, a^{\prime} a^{\prime \prime}}\left\langle\left. 0\right|_{g} V_{\epsilon ; a^{\prime} a}^{\dagger-} A^{a^{\prime} a^{\prime \prime}} V_{\epsilon ; a a^{\prime \prime}}^{-} \mid 0\right\rangle_{g}= \\
& =\sum_{a}\left\langle\left. 0\right|_{g}\left(A^{a a}+\sum_{a^{\prime} a^{\prime \prime}} V_{\epsilon ; a^{\prime} a}^{\dagger-}\left[V_{\epsilon ; a a^{\prime \prime}}^{-}, A^{a^{\prime} a^{\prime \prime}}\right]\right) \mid 0\right\rangle_{g} .
\end{aligned}
$$

В отсутствие регуляризации коммутатор $V_{\epsilon ; a a^{\prime}}^{ \pm}$с наблюдаемыми равен формально нулю. На регуляризованном уровне он отличен от нуля, но содержит положительные степени $\epsilon$, что приводит к подавлению инфракрасных расходимостей. Таким образом, в формуле (2.15) будут присутствовать только неглавные расходимости. 
Как известно, матричный элемент $S_{\epsilon}$ между чисто кварковыми состояниями имеет иерархическую структуру инфракрасных расходимостей вида

$$
S_{0}\left(\frac{g^{2}}{2 \epsilon}\right)+g^{2} S_{1}\left(\frac{g^{2}}{2 \epsilon}\right)+\cdots .
$$

Первое слагаемое ряда (2.16) описывает главные расходимости. Для него можно получить явное выражение

$$
\begin{aligned}
& \left\langle\left. 0\right|_{g} \otimes\left\langle\left.\Psi_{f}^{a}\right|_{q} S_{\epsilon} \mid \Psi_{i}^{b}\right\rangle_{q} \otimes \mid 0\right\rangle_{g}=\sum_{a^{\prime}, b^{\prime}}\left\langle\left. 0\right|_{g} V_{\epsilon ; a^{\prime} a}^{\dagger+} V_{\epsilon ; b b^{\prime}}^{-} \mid 0\right\rangle_{g} \times \\
& \times\left\langle\left. 0\right|_{g} \otimes\left\langle\left.\Psi_{f}^{a^{\prime}}\right|_{q} S_{\epsilon}^{\text {as }} \mid \Psi_{i}^{b^{\prime}}\right\rangle \otimes \mid 0\right\rangle_{g}+\text { (неглавные члены). }
\end{aligned}
$$

Формула (2.17) дает обобшение свойства “экспоненциации” инфракрасных расходимостей КХД. Отметим как важный для дальнейшего факт, что выражение

$$
\left\langle\left. 0\right|_{g} V_{\epsilon ; a a^{\prime}}^{\dagger+} V_{\epsilon ; b b^{\prime}}^{-} \mid 0\right\rangle_{g}
$$

является в “главном логарифмическом пределе"

$$
g \rightarrow 0, \quad \epsilon \rightarrow 0, \quad \frac{g^{2}}{2 \epsilon}=\lambda
$$

решением уравнений Киношиты-Укавы.

Теперь мы можем сформулировать рецепт устранения инфракрасных расходимостей из $S$-матрицы. Он заключается в переходе к нефоковским пространствам асимптотических состояний. Выбор $\left|\Psi_{i}^{\text {as }}\right\rangle=V_{\epsilon}^{\dagger-}\left|\Psi_{i}^{F}\right\rangle$ в качестве начального состояния и $\left|\Psi_{f}^{\text {as }}\right\rangle=V_{\epsilon}^{\dagger+}\left|\Psi_{f}^{F}\right\rangle$ в качестве конечного, где $\left|\Psi_{i, f}^{F}\right\rangle$ - состояния из фоковского пространства, устраняет инфракрасные расходимости из $S$-матрицы. Действительно, величина

$$
\left\langle\Psi_{f}^{\mathrm{as}}\left|S_{\epsilon}\right| \Psi_{i}^{\mathrm{as}}\right\rangle=\left\langle\Psi_{f}^{F}\left|S_{\epsilon}^{\mathrm{as}}\right| \Psi_{i}^{F}\right\rangle
$$

уже не содержит инфракрасных расходимостей в силу предполагаемых свойств асимптотической $S$-матришы.

\section{3. УСЛОВИЕ САМОСОГЛАСОВАННОСТИ ПОСТАНОВКИ ЗАДАЧИ РАССЕЯНИЯ И АСИМПТОТИЧЕСКИЕ СВОЙСТВА КВАРКОВ}

Использование представления асимптотической динамики подразумевает, что собственные состояния асимптотического гамильтониана образуют базис в пространстве асимптотических состояний $\mathcal{H}_{\text {as }}$. Если в $\mathcal{H}_{\text {as }}$ есть подпространство $\mathcal{H}_{\mathrm{as}}^{q}$, содержашее физические "инфракрасно одетые" кварки, и если последние могут быть получены из затравочных кварковых полей по теории возмушений, то операторы асимптотической эволюции реализуют унитарную эквивалентность пространства $\mathcal{H}_{\text {as }}^{q}$ и фоковского пространства “голых" кварков $\mathcal{F}_{q} \otimes|0\rangle_{g}$ :

$$
\mathcal{H}_{\mathrm{aS}}^{q}=V_{\epsilon}^{+} \mathcal{F}_{q} \otimes|0\rangle_{g} .
$$


Предположение о сушествовании физических кварков приводит, таким образом, при учете условия $(2.4)$ к требованию унитарности на пространстве $\mathcal{F}_{q}$ оператора

$$
M=\left\langle\left. 0\right|_{g} V_{\epsilon}^{\dagger+} V_{\epsilon}^{-} \mid 0\right\rangle_{g}
$$

Если же физические кварки могут быть получены по теории возмушений из голых кварков, принадлежаших некоторому подпространству фоковского пространства

$$
\mathcal{H}_{q}=P \mathcal{F}_{q}
$$

где $P$ - проектор, то $M$ должен быть унитарен на подпространстве $\mathcal{H}_{q}$, т.е.

$$
M P M^{\dagger} P=M^{\dagger} P M P=P
$$

Заметим, что в КЭД оператор $M$ имеет вид

$$
M_{\text {КЭД }}=\exp (2 i \lambda \Phi)
$$

где оператор кулоновской фазы

$$
\Phi=\frac{1}{8 \pi} \int \frac{d \vec{p}}{2 E_{\vec{p}}} \frac{d \vec{q}}{2 E_{\vec{q}}} \rho_{\mathrm{el}}(\vec{p}) \rho_{\mathrm{el}}(\vec{q}) \frac{p q}{\sqrt{(p q)^{2}-m^{4}}}
$$

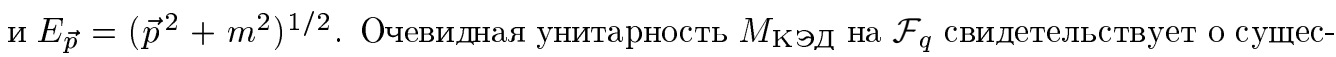
твовании в КЭД физических электронов.

Переходя к КХД, отметим, что инфракрасные расходимости коэффициентных функций оператора $M$ имеют ту же структуру, что и ряд (2.16). Легко показать, переходя к логарифмическому пределу (2.19), что оператор $M_{0}(\lambda)$, описывающий главные инфракрасные расходимости, должен быть сам по себе унитарен на пространстве $\mathcal{F}_{q}$. Уравнение (1.1) может быть записано для $M_{0}(\lambda)$ в следуюшем виде:

$$
\begin{aligned}
& \frac{d M_{0}(\lambda)}{d \lambda}=f(\lambda)\left(\int_{S^{2}} \frac{d \hat{\vec{k}}}{4 \pi} \int \frac{d \vec{p}}{2 E_{\vec{p}}} \frac{d \vec{q}}{2 E_{\vec{q}}} \frac{p q}{(p \hat{k})(q \hat{k})} \times\right. \\
& \left.\quad \times\left[\rho^{a}(\vec{p}),\left[\rho^{a}(\vec{q}), M_{0}(\lambda)\right]\right]+i\left\{\Phi_{\mathrm{KХ} Д}, M_{0}(\lambda)\right\}\right) .
\end{aligned}
$$

Здесь $\hat{\vec{k}}$ - вектор на единичной сфере в трехмерном пространстве. Неабелев аналог ку-

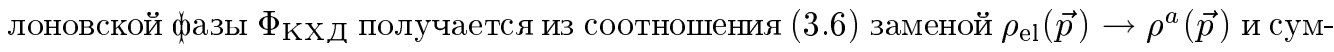
мированием по $a$,

$$
f(\lambda)=\left(1-\frac{22}{3} C_{A} \frac{\lambda}{(4 \pi)^{2}}\right)^{-1} .
$$

Покажем, что явньй вид функции $f(\lambda)$ несушествен для дальнейшего. 
TеOPEMA 1. Пусть оператор

$$
M(x)=\sum_{n=0}^{\infty} M_{n} x^{n}, \quad M_{0}=1,
$$

удовлетворяет условию унитарности во всех порядках по $x$, m.e.

$$
\sum_{k=0}^{n} M_{n-k}^{\dagger} M_{k}=0 \quad \forall n \geqslant 1 .
$$

Тогда оператор

$$
\widetilde{M}=M(f(x))
$$

əде

$$
f(x)=\sum_{n=1}^{\infty} f_{n} x^{n}, \quad f_{1}=1,
$$

также унитарен (в смысле разложсения по $x$ ).

ДокаЗАТЕльСтво. Разложим $\widetilde{M}^{\dagger}(x) \widetilde{M}(x)$ в ряд по $x$ с учетом (3.12). В $n$-м порядке получим выражение

$$
\sum_{k=0}^{n} \widetilde{M}_{n-k}^{\dagger} \widetilde{M}_{k}=\sum_{q=1}^{n} \sum_{\substack{\gamma_{1}, \ldots, \gamma_{q} \\ \sum \gamma_{q}=n}} f_{\gamma_{1}} \ldots f_{\gamma_{q}}\left(\sum_{l=0}^{q} M_{q-l}^{\dagger} M_{l}\right)
$$

которое делает унитарность $\widetilde{M}$ очевидным следствием условия (3.10).

Таким образом, явный вид зависимости эффективного заряда от $\lambda$ несуществен для условия самосогласованности постановки задачи рассеяния. В связи с этим мы будем считать, что $f(\lambda)=1$.

Сделаем некоторые удобные переобозначения. Вместо $M_{0}(\lambda)$ будем писать

$$
M(\lambda)=\sum_{n=0}^{\infty} M_{n} \lambda^{n}
$$

где $M_{0}=I$. Вместо интеграла

$$
\int_{S^{2}} \frac{d \hat{\vec{k}}}{4 \pi} \int \frac{d \vec{p}}{2 E_{\vec{p}}} \frac{d \vec{q}}{2 E_{\vec{q}}} \frac{p q}{(p \hat{k})(q \hat{k})} \rho^{a}(\vec{p}) \ldots \rho^{a}(\vec{q})
$$

будем писать

$$
\widehat{\rho \ldots \rho},
$$

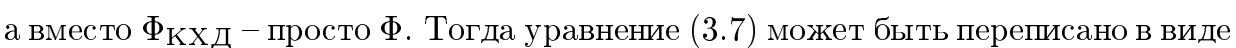

$$
\begin{aligned}
& \left.n M_{n}=\left[\widehat{\rho,[\rho}, M_{n-1}\right]\right]+i\left\{\Phi, M_{n-1}\right\}, \\
& M_{0}=I .
\end{aligned}
$$


Отсюда можно найти $M_{n}$ для любого наперед заданного номера $n$.

Из условий унитарности (3.4) можно получить следуюшие следствия для $n \geqslant 1$ :

$$
\begin{aligned}
& {\left[M_{n}, P\right]=0, \quad\left[M_{n}^{\dagger}, P\right]=0,} \\
& P \sum_{k=0}^{n} M_{k} M_{n-k}^{\dagger} P=P \sum_{k=0}^{n} M_{k}^{\dagger} M_{n-k} P=0 .
\end{aligned}
$$

Вычислим теперь явно $M_{1}, M_{2}$ и $M_{3}$, используя $(3.15)$ :

$$
\begin{aligned}
& M_{1}=2 i \Phi, \\
& \left.M_{2}=i[\widehat{\rho,[\rho}, \Phi]\right]-2 \Phi^{2} \\
& \left.\left.\left.\left.\left.M_{3}=\frac{i}{3}[\widehat{\rho,[\rho}, \widehat{[\rho,[\rho}, \Phi]\right]\right]\right]-\frac{2}{3}\left[\widehat{\rho,[\rho}, \Phi^{2}\right]\right]-\frac{1}{3}\{[\widehat{\rho,[\rho}, \Phi]], \Phi\right\}-\frac{4}{3} i \Phi^{3} .
\end{aligned}
$$

Подставляя $M_{1}$ из $(3.18)$ в (3.16), получим

$$
[\Phi, P]=0
$$

Подставляя $M_{1}, M_{2}$ и $M_{3}$ из $(3.18)$ в $(3.17)$, имеем

$$
P[\Phi, \widehat{\rho],[\rho}, \Phi] P=0 .
$$

Из условия (3.20) и положительности “спаривания" (3.14) следует, что

$$
[\rho, \Phi] P=0 .
$$

Можно доказать теорему, обобщающую это условие на все порядки.

ТЕОРема 2. Для любого п выполняется соотношение

$$
[\rho, \Phi] \underbrace{\rho \ldots \rho}_{n} P=0 .
$$

ДОКАЗАТЕЛЬСТВО этой теоремы проводится по индукции. С помощью уравнения (3.15) рекуррентным путем получается следуюшее выражение для $M_{n}$, записанное в форме ряда по $\Phi$ :

$$
\begin{aligned}
M_{n}= & 2\left(\frac{i(-1)^{n-1}}{(2 n-1) !}\left\langle[\rho, \Phi]_{2 n-2}\right\rangle+\right. \\
& \left.+(-1)^{n-1} \sum_{k=0}^{2 n-4} \frac{\left\langle[\rho, \Phi]_{k}[\rho, \Phi]_{2 n-k-4}\right\rangle}{(k+1) !(2 n-k-3) !}+\cdots+i \frac{(2 i)^{n-1}}{n !} \Phi^{n}\right)
\end{aligned}
$$

где

$$
[\rho, \Phi]_{n}=[\rho, \ldots,[\rho, \underbrace{\Phi] \ldots]}_{n}
$$


- составной коммутатор, а знак 〈〉 обозначает суммирование по всем возможным спариваниям операторов $\rho$ друг с другом (возможны спаривания либо операторов $\rho$, принадлежащих к различным составным коммутаторам, либо операторов, входящих в один составной коммутатор и стоящих рядом).

Из (3.21) и предположения индукции следует, что

$$
\begin{aligned}
M_{n} P= & P M_{n} P=\frac{(2 i \Phi)^{n}}{n !} P, \quad n=0,1, \ldots, N \\
M_{N+1}= & \frac{2 i^{2 N+1}}{(2 N+1) !} P\left\langle[\rho, \Phi]_{2 N}\right\rangle P+\frac{(2 i \Phi)^{N+1}}{(N+1) !} P \\
M_{N+2}= & \frac{2 i^{2 N+3}}{(2 N+3) !} P\left\langle[\rho, \Phi]_{2 N+2}\right\rangle P+ \\
& +2 i^{2 N} \sum_{k=0}^{2 N} \frac{1}{(2 N-k+1) !(k+1) !} P\left\langle[\rho, \Phi]_{2 N-k}[\rho, \Phi]_{k}\right\rangle P+ \\
& +\frac{1}{(N+2) !}(2 i \Phi)^{N+2} P .
\end{aligned}
$$

В последней сумме в силу предположения индукции отличен от нуля лишь член с $k=N$. Подстановка (3.24) в условие (3.17) для $n=N+2$ дает условие

$$
P\left\langle\rho^{N-1}[\rho, \Phi]_{1}[\Phi, \rho]_{1} \rho^{N-1}\right\rangle P=0
$$

Теперь нужно учесть, что в силу предположения индукции оператор

$$
\left[\Phi, \rho^{a_{0}}\left(\vec{p}_{0}\right)\right] \rho^{a_{1}}\left(\vec{p}_{1}\right) \ldots \rho^{a_{N}}\left(\vec{p}_{N}\right) P
$$

не меняется при перестановках операторов $\rho^{a_{i}}\left(\vec{p}_{i}\right)$ и потому симметричен как функция $\left\{\vec{p}_{i}, a_{i}\right\}$. Действительно, операторы $(3.26)$ с различным порядком $\rho^{a_{i}}\left(\vec{p}_{i}\right)$ равны друг другу с точностью до выражений, в которых некоторые пары заменены их коммутаторами. Однако замена пары операторов $\rho$ коммутатором приводит к уменьшению обшего числа операторов $\rho$ в операторе (3.26) на единицу, что в силу предположения индукции обрашает в нуль такие выражения. Вследствие симметрии оператора (3.26) можно так расположить операторы $\rho^{a_{i}}\left(\vec{p}_{i}\right)$ в условии $(3.25)$, что спаривания между ними будут симметричны по отношению к оператору $[\rho, \Phi][\Phi, \rho]$ в (3.25). После этого из положительности спаривания следует, что

$$
[\Phi, \rho] \underbrace{\rho \ldots \rho}_{N-1} P=0,
$$

что и требовалось доказать.

Легко видеть, что верна и обратная теорема.

Таким образом совокупность условий (3.22) эквивалентна требованию унитарности оператора $M$ на пространстве физических состояний кварков.

Теперь мы сформулируем теорему, непосредственно описывающую условия, которым должны удовлетворять физические состояния. 
ТЕОРема 3. Пусть дан набор конечномерных $(k=1, \ldots, N)$ пространств $\mathcal{L}_{k}$, в каждом из которых определено несинглетное унитарное представление простой компактной группь $G$ с генераторами $I_{k}^{a}$. Рассмотрим в прямом произведении этих пространств

$$
\mathcal{L}=\bigotimes_{k=1}^{N} \mathcal{L}_{k}
$$

onepamop

$$
\Phi_{N}=\sum_{k, m} I_{k}^{a} I_{m}^{a} R_{k m}
$$

əде $R_{k m}=R_{m k}, R_{k k}=0$.

Пусть существует подпространство $\mathcal{L}_{0} \subset \mathcal{L}$ такое, что

$$
\left[\Phi_{N}, I_{k_{0}}^{a_{0}}\right] I_{k_{1}}^{a_{1}} \ldots I_{k_{p}}^{a_{p}} \mathcal{L}_{0}=0
$$

для любого набора $\left\{a_{i}, k_{i}\right\}$. Тогда либо $\mathcal{L}_{0}$ тривиально, либо $R_{k m}=0$ при всех $k, m$.

ДокАЗАТЕЛЬство. Введем в пространстве $\mathcal{L}_{k}$ ортонормированный базис $\left\{\left|\alpha_{k}\right\rangle\right\}$. Тогда произвольное состояние $|\Psi\rangle \in \mathcal{L}$ можно представить в виде

$$
|\Psi\rangle=\sum_{\alpha_{1}, \ldots, \alpha_{N}} \Psi^{\alpha_{1}, \ldots, \alpha_{N}}\left|\alpha_{1}\right\rangle \otimes \cdots\left|\alpha_{N}\right\rangle=\sum_{\alpha_{1}}\left|\alpha_{1}\right\rangle \otimes\left|\Psi^{\alpha_{1}}\right\rangle .
$$

Легко видеть, что условие (3.29) эквивалентно условию

$$
\Phi_{N} F[I] \mathcal{L}_{0}=0
$$

для любого полинома $F[I]$.

Пусть $F$ зависит только от $I_{1}$. Для $|\Psi\rangle \in \mathcal{L}_{0}$ и произвольного вектора

$$
|\widetilde{\Psi}\rangle \in \mathcal{L}^{\prime}=\bigotimes_{2}^{N} \mathcal{L}_{k}
$$

вычислим

$$
\left\langle\widetilde{\Psi}\left|\Phi_{N} F\left[I_{1}\right]\right| \Psi\right\rangle=\left\langle\widetilde{\Psi}\left|F\left[I_{1}\right] \Phi_{N}\right| \Psi\right\rangle .
$$

Используя для $|\Psi\rangle$ представление (3.30), получим

$$
\sum_{a} I_{1}^{a} F\left[I_{1}\right]\left|\phi^{a}\right\rangle=\sum_{a} F\left[I_{1}\right] I_{1}^{a}\left|\phi^{a}\right\rangle
$$

где

$$
\left|\phi^{a}\right\rangle=2 \sum_{\alpha_{1}}\left|\alpha_{1}\right\rangle\left\langle\widetilde{\Psi}\left|\sum_{n} R_{1 n} I_{n}^{a}\right| \Psi^{\alpha_{1}}\right\rangle .
$$

Нетрудно показать, что условие (3.31) эквивалентно условию

$$
\left[I_{1}^{a}, I_{1}^{b_{0}}\right] I_{1}^{b_{1}} \ldots I_{1}^{b_{n}}\left|\phi^{a}\right\rangle=0
$$


для любого набора $\left\{b_{i}\right\}$. Отсюда имеем, что

$$
\left(I_{1}^{b} I_{1}^{a} I_{1}^{b}-I_{1}^{b} I_{1}^{b} I_{1}^{a}\right)\left|\phi^{a}\right\rangle=0,
$$

но

$$
I_{1}^{b} I_{1}^{b}=c_{1} I
$$

a

$$
\begin{aligned}
I_{1}^{b} I_{1}^{a} I_{1}^{b} & =I_{1}^{b} I_{1}^{b} I_{1}^{a}+\frac{1}{2} i\left(f^{a b c} I_{1}^{b} I_{1}^{c}+f^{a c b} I_{1}^{c} I_{1}^{b}\right)=c_{1} I_{1}^{a}+\frac{1}{2} i f^{a b c}\left(I_{1}^{b} I_{1}^{c}-I_{1}^{c} I_{1}^{b}\right)= \\
& =c_{1} I_{1}^{a}+\frac{1}{2} i f^{a b c} f^{b c d} I_{1}^{d}=c_{1} I_{1}^{a}-\frac{1}{2} c_{A} \delta^{a d} I_{1}^{d}=c_{1} I_{1}^{a}-\frac{1}{2} c_{A} I_{1}^{a},
\end{aligned}
$$

где $c_{1}$ - значение квадратичного оператора Казимира представления группы $G$ в пространстве $\mathcal{L}_{1}$, а $c_{A}$ - значение этого же оператора в присоединенном представлении. Подставляя (3.34) и (3.35) в (3.33), получим

$$
\left(\left(c_{1}-\frac{1}{2} c_{A}\right) I_{1}^{a}-c_{1} I_{1}^{a}\right)\left|\phi^{a}\right\rangle=-\frac{1}{2} c_{A} I_{1}^{a}\left|\phi^{a}\right\rangle=0,
$$

откуда

$$
I_{1}^{a}\left|\phi^{a}\right\rangle=0 .
$$

Следовательно, условие (3.31) имеет вид

$$
\sum_{a} I_{1}^{a} F\left(I_{1}^{a}\right)\left|\phi^{a}\right\rangle=0
$$

для любого $F\left(I_{1}\right)$.

Выберем в пространстве $\mathcal{L}_{1}$ произвольное состояние $|\xi\rangle$ и положим

$$
M^{b a}(\xi) \equiv\left\langle\xi \mid I_{1}^{b} \phi^{a}\right\rangle .
$$

Легко показать, что (3.36) эквивалентно условию

$$
\operatorname{tr}(M(\xi) \Lambda)=0
$$

где $\Lambda$ - произвольный оператор в пространстве присоединенного представления группы $G$, являюшейся полиномом от его генераторов. В силу шикличности присоединенного представления простой группы $G$ из (3.37) следует, что

$$
M^{b a}(\xi)=0 \quad \forall \xi \in \mathcal{L}_{1} .
$$

Отсюда заключаем, что $\left|\phi^{a}\right\rangle=0$ и вследствие произвольности $|\widetilde{\Psi}\rangle$ имеем

$$
\sum_{n} R_{1 n} I_{n}^{a}|\Psi\rangle=0 \quad \forall|\Psi\rangle \in \mathcal{L}_{0} .
$$


Подставим теперь в уравнение (3.38) вектор $I_{k}^{b}|\Psi\rangle$ вместо вектора $|\Psi\rangle(k \neq 1)$, подействуем на это состояние оператором $I_{k}^{b}$, просуммируем по $b$, а затем вычтем из полученного результата уравнение (3.38), умноженное на $c_{k}$. При этом будем иметь

$$
R_{1 k} I_{k}^{a}|\Psi\rangle=0
$$

для любого $a$, что и доказывает теорему.

Применим теперь эту теорему к рассмотрению пространства асимптотических состояний. Рассмотрим собственное подпространство оператора плотности числа частиц, содержашее $N$ частиц с импульсами $\vec{p}_{1}, \ldots, \vec{p}_{N}$. С точки зрения группы симметрии это подпространство представляет собой пространство вида (3.27), а оператор кулоновской фазы $\Phi$ сводится к оператору (3.28) с коэффициентами

$$
R_{k m}=R\left(\vec{p}_{k}, \vec{p}_{m}\right),
$$

которые равны нулю лишь при $\vec{p}_{k}=\vec{p}_{m}$ (это следует из свойств кулоновской фазы). Поэтому допустимое состояние $|\Psi\rangle$ массивных частиц должно удовлетворять условию

$$
\rho^{a}(\vec{p})|\Psi\rangle=I^{a} \delta\left(\vec{p}-\vec{p}_{0}\right)|\Psi\rangle,
$$

где

$$
I^{a}=\int \frac{d \vec{p}}{2 E_{\vec{p}}} \rho^{a}(\vec{p})
$$

- оператор цветного заряда, $\vec{p}_{0}$ - некоторый импульс, характерный для данного состояния.

Выполнение условия (3.39) для вакуумного и одночастичного состояний гарантируется аксиомами $S$-матричного подхода. В то же время никакие другие фоковские состояния кварков условию (3.39) не удовлетворяют (действительно, состояние из двух частиц с совпадающими импульсами является ненормируемым и потому не принадлежит фоковскому пространству). Это означает отсутствие фоковских состояний, соответствующих системе разлетающихся кварков.

Приведенные выше результататы могут быть формально обобщены на случай связанных состояний. В этом случае условие унитарности оператора $M$ выполняется лиш для бесцветных состояний. Здесь, однако, нельзя говорить о строгих доказательствах, поскольку мы оказываемся за пределами применимости теории возмущений.

Следует отметить, что физическая идея, лежащая в основе нашего подхода, проста. Суть ее сводится к тому, что в теории с безмассовыми частицами можно избавиться от инфракрасных расходимостей, перейдя к асимптотическим состояниям, в которых исходные фоковские состояния заменяются состояниями, близкими к исходным по суммарному импульсу и энергии и совпадающими с ними по дискретным квантовым числам. Однако в КХД (и в других неабелевых теориях с массивными фермионами и безмассовыми бозонами $[22,23])$ построить такие состояния с помощью унитарного оператора асимптотической эволюции невозможно.

Отметим также, что из двух факторов, обусловливающих усложнение инфракрасной структуры КХД по сравнению с КЭД, для нас оказался существенным только один неабелевость кварк-глюонного взаимодействия. Второй же фактор - самодействие безмассовых глюонов - не сыграл никакой роли в получении ограничений на асимптотические состояния массивных кварков в КХД. 


\section{Список литературы}

[1] A. Yu. Kamenshchik, N. A. Sveshnikov. Phys. Lett. B. 1983. V. 123. P. 255.

[2] L. Lyons. Phys. Rep. 1985. V. 129. P. 225.

[3] H. Pagels. Phys. Rev. D. 1976. V. 14. P. 2747; A. R. Swift, F. S. Roig. Phys. Rev. D. 1978. V. 18. P. 1206.

[4] M. Bander. Phys. Rep. 1981. V. 75. P. 205.

[5] Б. А. Арбузов, С. С. Куренной. ЯФ. 1982. Т. 36. С. 1314; А. И. Алексеев, Б. А. Арбузов, B. А. Байков. ЯФ. 1981. Т. 34. С. 1374; A. A. Славнов. ТМФ. 1983. Т. 54. С. 52; J. S. Ball, F. Zachariazen. Nucl. Phys. B. 1978. V. 143. P. 148; M. Baker, J.S. Ball, P. Lucht, F. Zachariazen. Phys. Lett. B. 1979. V. 86. P. 52.

[6] T. Appelquist, J. Carazzone. Nucl. Phys. B. 1977. V. 120. P. 77; E. C. Poggio, H. R. Quinn. Phys. Rev. D. 1976. V. 14. P. 578.

[7] T. Kinoshita, A. Ukawa. Phys. Rev. D. 1977. V. 15. P. 1596.

[8] T. Kinoshita, A. Ukawa. Phys. Rev. D. 1977. V. 16. P. 332.

[9] R. Doria, J. Frenkel, J. C. Taylor. Nucl. Phys. B. 1980. V. 168. P. 93; C. Di'Ideto, S. Gendron, I. G. Hallidey, C. T. Sachrajda. Nucl. Phys. B. 1981. V. 183. P. 223.

[10] L. Tyburski. Nucl. Phys. B. 1976. V. 116. P. 214; G. Sterman. Phys. Rev. D. 1976. V. 14. P. 2123.

[11] D. Shalloway. Phys. Rev. D. 1979. V. 19. P. 1762; C.E. Carneiro, M. Day, J. Frenkel, J. C. Taylor. Nucl. Phys. B. 1981. V. 183. P. 445; A. Andrasi, M. Day, R. Doria, J. Frenkel, J. C. Taylor. Nucl. Phys. B. 1981. V. 182. P. 104.

[12] T. M. Cornwall, G. Tiktopoulos. Phys. Rev. D. 1976. V. 13. P. 3370.

[13] П. П. Кулии, Л. Д. Фаддеев. ТМФ. 1970. Т. 4. С. 153.

[14] D. R. Butler, C. A. Nelson. Phys. Rev. D. 1978. V. 18. P. 1196; C. A. Nelson. Nucl. Phys. B. 1981. V. 181. P. 141; V. 186. P. 187.

[15] J. Frenkel, J. G. M. Gatheral, J. C. Taylor. Nucl. Phys. B. 1982. V. 194. P. 172.

[16] D. R. Yennie, S. C. Frautschi, H. Suura. Ann. Phys. 1961. V. 13. P. 379.

[17] T. Kinoshita. J. Math. Phys. 1962. V. 3. P. 650; T.D. Lee, M. Nauenberg. Phys. Rev. 1964. V. 133. P. 1549.

[18] F. Bloch, A. Nordsiek. Phys. Rev. 1937. V. 52. P. 54.

[19] S. B. Libby, G. Sterman. Phys. Rev. D. 1979. V. 19. P. 2468.

[20] W. Magnus. Commun. Pure Appl. Math. 1954. V. 7. P. 649.

[21] Н. А. Свешников. Пространство асимптотических состояний в теории $\bar{\Psi} \Psi$. Препринт Р2-12267. Дубна: ОИЯИ, 1979.

[22] Н. А. Свешников. Асимптотическая динамика в зарядово-симметричной модели. Препринт Р2-12862. Дубна: ОИЯИ, 1979.

[23] Н. А. Свешников. Пространство асимптотических состояний в зарядово-симметричной модели. В сб.: Труды международного семинара "Проблемы физики высоких энергий и квантовой теории поля", 20-27 сентября 1980 г. Серпухов: ИФВЭ, 1980. Т. 1. С. 116.

[24] G. t'Hooft, M. Veltman. Nucl. Phys. B. 1972. V. 44. P. 189; C. G. Bollini, J. J. Giambiagi. Nuovo Cimento. 1972. V. 12. P. 20; J. Ashmore. Lett. Nuovo Cimento. 1972. V. 4. P. 289; C. M. Cicuta, E. Montaldi. Lett. Nuovo Cimento. 1972. V. 4. P. 329.

Поступила в редакцию $14 . \mathrm{IV} .1998$ г. 